\title{
The role of ultrasonography in determination of the appropriate endotracheal tube size selection in pediatric patients
}

\author{
Altun D., Sivrikoz N., Ali A., Orhan Sungur M., Salviz A.E., Çamc1 E. \\ Istanbul University, Istanbul Faculty of Medicine, Department of Anesthesiology and Perioperative Medicine, Turkey
}

\section{Background and Goal of Study:}

The aim of this prospective clinical study was to investigate the success of ultrasonography (USG) in pediatric patients in predicting the appropriate sized cuffed ETT and compare the results with aged and Broselow tape formulas.

\section{Materials and methods:}

- Children aged between 0-13 years who received general anesthesia with endotracheal intubation were enrolled in the study.

- In all participants, subglottic airway diameter was measured with USG (Figure 1) and Motoyama-Khine and Broselow tape ETT size was calculated.

- For USG method, transverse diameter of subglottic airway was measured at the anterior neck at the caudal of the cricoid cartilage without ventilation. ETT size was chosen according to the measured subglottic airway diameter. If there was resistance to passage of the tube into the trachea, a tube one size smaller was replaced. Airway pressures (P) were recorded and if $\mathrm{P}$ was above $25 \mathrm{cmH}_{2} 0$, ETT was replaced with one size larger.

- Need for ETT replacement were recorded.

- A Bland-Altman assessment was used for assess the similarity of two measurement method.

\section{Results:}

- 120 pediatric patients were included. Demographic data are presented in Table 1.

- USG measured diameter was found to be significantly smaller than the diameter as measured by other methods. According to Bland-Altman analysis, measurement with USG was significantly different from age-based calculation $(\mathrm{p}<0.001)$. Likewise, measurement with USG was also significantly different from Broselow tape calculation( $\mathrm{p}<$ 0.001).(Figure 2, 3)

- In eights patients, ETT was changed with a one size larger tube and therefore success rate with USG was \%93.3. The average peak pressure was $17.7 \pm 3.0 \mathrm{~cm} \mathrm{H}_{2} 0$.

\section{Discussion:}

In this study, USG measurement guided ETT size selection resulted in smaller diameter tubes when compared to Motoyama-Khine and Broselow tape calculations. Out of the two formulas, Broselow tape based calculation was more closely related with USG measurements.

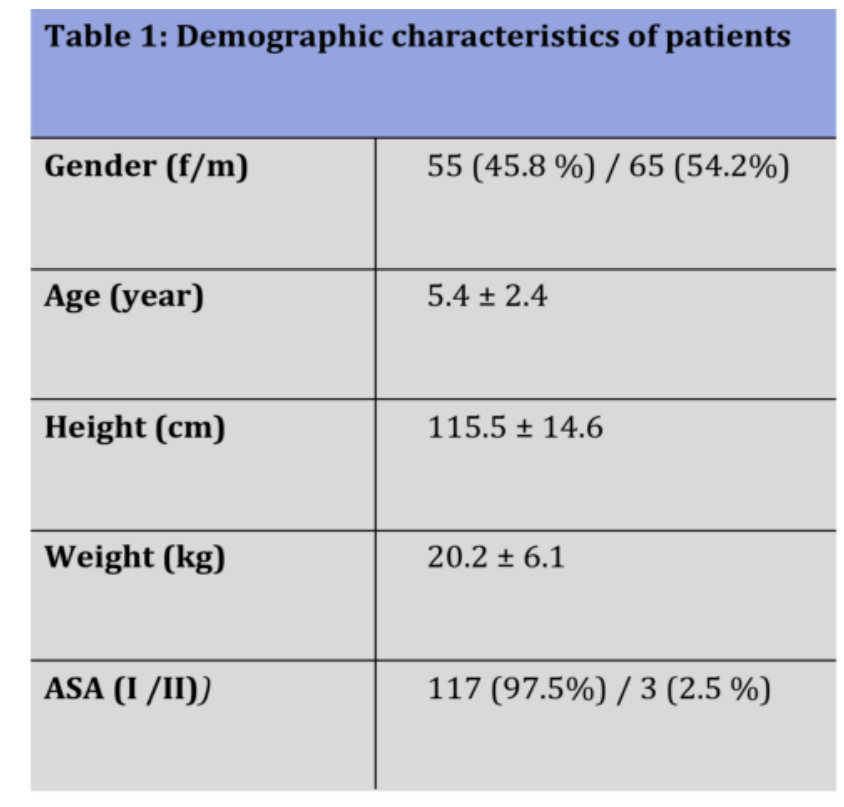

Figure 1: Ultrasonographic measurement of transverse diameter

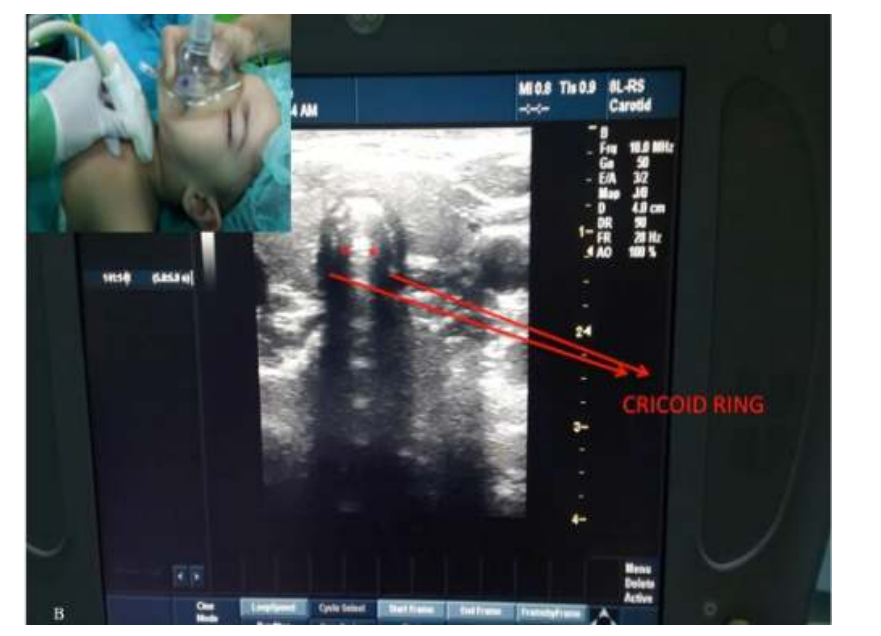

Figure 2: Blant-Altman analysis of the diameter differences between USG measurement and Aged based formula

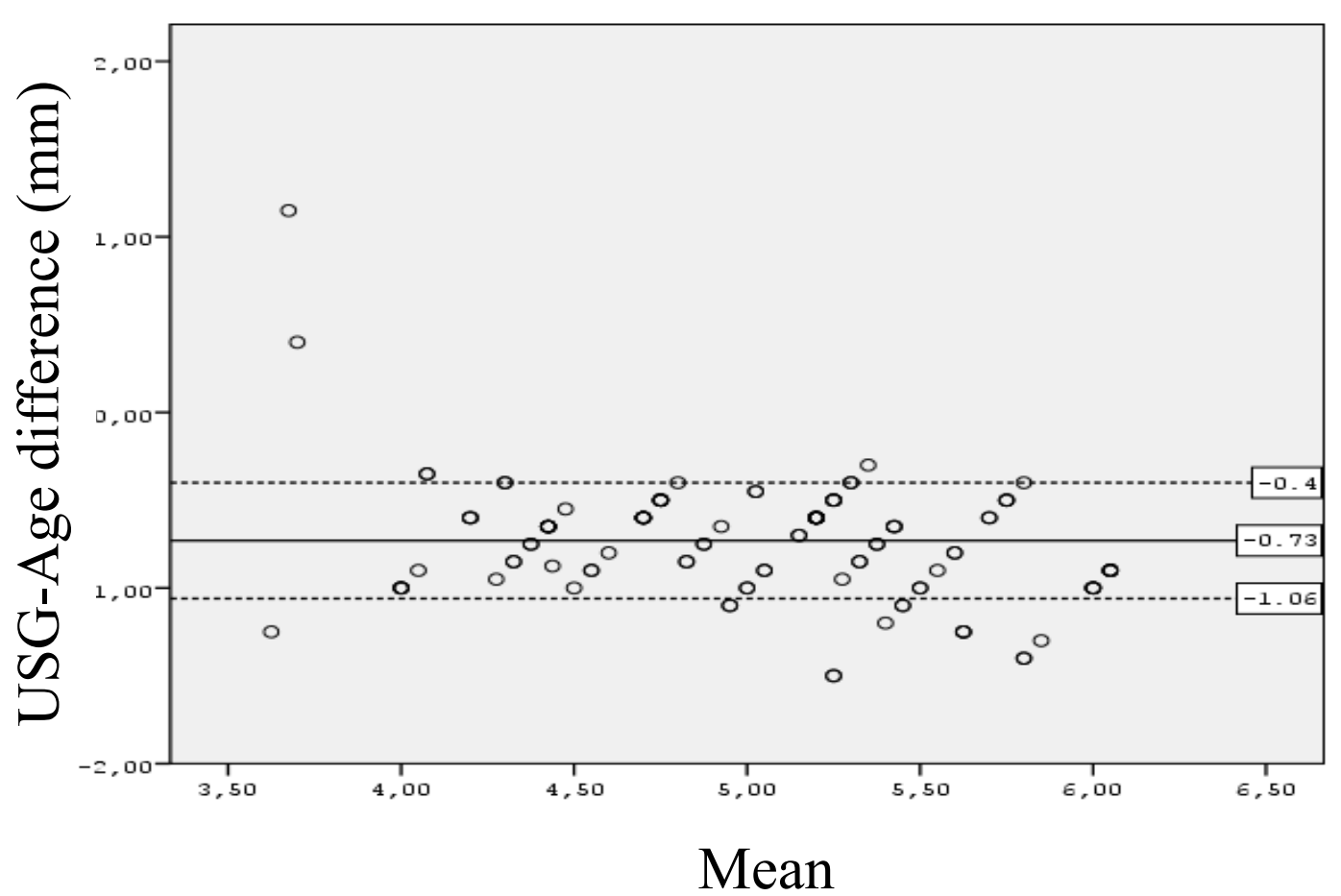

Figure 3: Blant-Altman analysis of the diameter differences between USG measurement and Broselow tape formulas

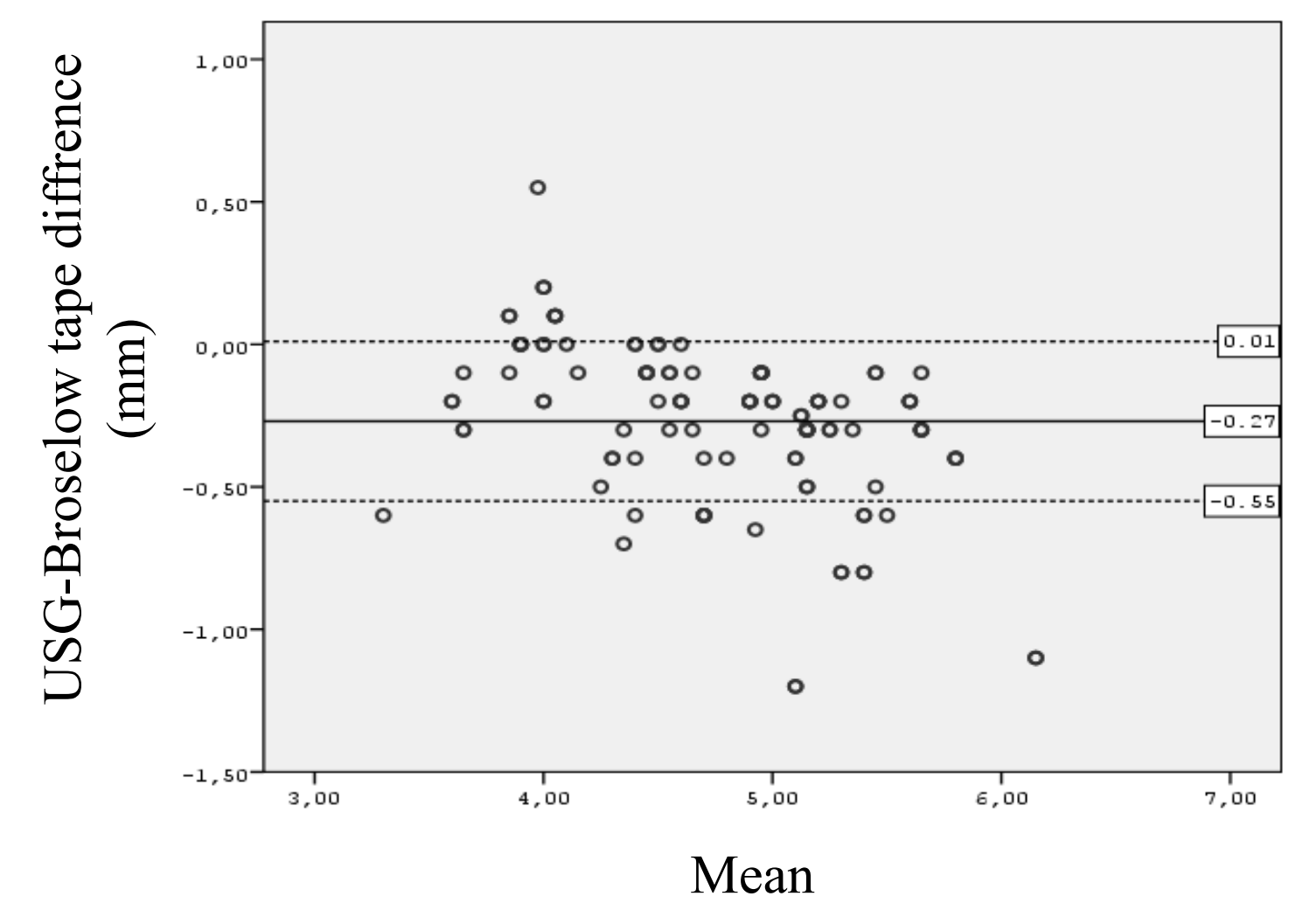

\section{Conclusion:}

- USG appears to be a reliable predictor for the assessment of the subglottic diameter of the airway in children to estimate the appropriate size ETT for intubation. 